\title{
Limitations of child injury data from the CPSC's National Electronic Injury Surveillance System: the case of baby walker related data
}

\author{
Harold B Weiss
}

\begin{abstract}
Objectives-The US Consumer Product Safety Commission's National Electronic Injury Surveillance System (NEISS) is a primary source for children's consumer product injury surveillance data in the US. Differing interpretations of the emergency department based NEISS baby walker data by various parties prompted this detailed examination, reclassification, and analysis of the NEISS data to explain these discrepancies.
\end{abstract}

Methods-Case selection was performed by searching the NEISS 1982-91 database for the baby walker product code and various text strings for children less than 24 months old. False negative and false positive cases were identified and reclassified. Adjusted population rates were computed and the types and locations of hospitals contributing to the sample were examined.

Results-One per cent false positive and $4 \%$ false negative misclassification rates were observed. In 1991, two children's hospitals reported $14 \%$ of the baby walker related injuries, though these hospitals made up just $2 \%$ of the sample frame. Through random allocation, one state currently contains four acute care hospitals and the only two children's hospitals reporting to the NEISS system. These six hospitals contributed $18 \%$ of the walker cases whereas the state represents only $3 \%$ of the US infant population.

Conclusions-Misclassification in NEISS baby walker reports is minimal, with false negatives outweighing false positives. For trend analysis of product related injuries at the frequency of occurrence observed for baby walkers, NEISS suffers from low sensitivity due to sampling error. For children's injuries, NEISS' estimates have been affected by children's hospitals coming in and out of the sample and currently reflects a random geographic imbalance because one state contributes both of the reporting children's hospitals. To overcome these problems improved multiple product coding, a unique baby walker code, and stratification of children's hospitals in an enlarged NEISS sample is recommended.

(Injury Prevention 1996; 2: 61-66)
Keywords: consumer product safety, emergency department, population surveillance, baby walkers.

Since 1972, the US Consumer Product Safety Commission (CPSC) National Electronic Injury Surveillance System (NEISS) has been a primary source for consumer product injury surveillance data. ${ }^{1}$ NEISS statistics are often quoted, used for scientific publications, ${ }^{2-4}$ receive widespread media coverage, and are used to initiate and develop CPSC regulatory actions, especially for child related products. While about $40 \%$ of all NEISS reports concern children ages $0-14$, in a recent one month period $77 \%$ (10 of 13 ) of CPSC press releases pertained to children and risks to children. ${ }^{5}$ Of all 1994 proposed CPSC regulations, 73\% (14 of 19) were targeted toward products of particular relevance to children. ${ }^{6}$

Baby walkers are one of these child related products that have received a great deal of attention by CPSC over the years. CPSC's involvement and the NEISS data itself have often been associated with widely contrasting epidemiologic interpretations of the baby walker data by proponents and opponents. These differing claims prompted this detailed examination of the data for possible explanations. The aim was to determine the usefulness and limitations of the NEISS data for baby walkers and by analogy, perhaps other child product related injuries.

Baby walker related injuries

A baby walker is a juvenile furniture device with a seat and leg openings usually mounted to a plastic deck attached to a base with wheels. Walkers are used to keep babies occupied and happy, though some parents mistakenly believe walkers assist a child in learning to walk. ${ }^{7}$ Their ownership and use in the US have become an icon of pretoddler activity. The annual US production of baby walkers is estimated at almost two million units per year by industry sources (unpublished data, Juvenile Products Manufacturers Association, personal communication, 1992) and in the published literature.$^{89}$ An estimated $70-90 \%$ of 6 to 12 months old children use a walker at some time. ${ }^{1011}$

Concomitant with the high use is an associated injury risk. Much of what is known about walker related injuries is based on the NEISS data, CPSC case reports, and a few 
small hospital based studies. In 1980, the NEISS reported about 10000 walker related injuries. By 1991, the NEISS estimates increased to $27800 .{ }^{12}$ In one hospital case series of clinic, emergency department, or hospitalized head injuries, walker related injuries represented the third most common mechanism of head injuries in children less than 2 years old. ${ }^{13}$

Most serious walker related trauma seen in the emergency department results from stair falls. ${ }^{12}$ In a survey of fall injuries at a Canadian children's hospital emergency department, only one baby walker injury was reported not related to stairs among 124 events. ${ }^{8}$ In addition to stair falls, the increased mobility and access provided by a walker are a factor in burns caused by pulling down hot liquids and in toxic exposures. ${ }^{14}$

Concern about the safety of baby walkers has been expressed for many years. This concern reached a high point in August 1992 with the submission of a petition to the CPSC by the Consumer Federation of America, American Academy of Pediatrics, National SAFE KIDS campaign, and the Consumers Union seeking their ban. Along with this petition came a series of claims and counter claims regarding the incidence and trends of baby walker related injuries using NEISS data. For example, in 1992 the Consumer Federation of America wrote: 'Each year as many as 28900 children are admitted in hospital emergency rooms with walker associated injuries'. A baby walker manufacturer claimed, however, that: ' $\ldots 23{ }^{\prime}$, of the NEISS [walker] numbers may represent doorway jumper incidents'. ${ }^{15}$ In another instance, a medical group reported: 'infant injuries from such walkers increased almost 45 per cent from 19997 in 1986 to 28913 in 1991'.16 In contrast, CPSC staff reported: 'There was no significant change in the rate of injury over the period 1984 to 1991 involving walkers' ${ }^{16}$ These contrasting claims prompted us to examine reasons for these discrepancies. We sought to clarify the surveillance methodology used by NEISS in order to determine the usefulness and limitations of the NEISS baby walker data.

\section{Methods}

THE NEISS SYSTEM

The NEISS collects a standardized data set from a national sample of emergency department patients treated for product related injuries. The data fields collected include date of treatment, age and sex of patient, injury diagnosis/body part, disposition, two product codes, third product (uncoded) or child resistant closure involvement, accident locale, fire or motor vehicle involvement, and two 71 character text comment fields. Patient data is obtained from emergency department records by a designated and trained NEISS coder through emergency department log and medical record review. Attempts are made by CPSC trainers to orient as many emergency department staff as possible to the need for documenting product involvement. After identification, reports are locally coded and electronically forwarded to a central location. NEISS cases are drawn from all (qualified) first visits that involve product related injuries seen in the sampled emergency departments. By legislative mandate the NEISS excludes injuries from most motor vehicles, drugs and cosmetics, alcohol, tobacco, and firearms.

The sample is designed to be statistically representative of all hospitals with emergency departments located within the US and its territories. As of January 1995, 91 hospitals contribute to NEISS ${ }^{17}$; however, the numbers and types of reporting hospitals have varied over the years. Estimates of national frequencies are achieved by applying a weighting factor specific to each observation in the sample taking into account the stratified (by number of visits) sampling frame and hospital specific circumstances. Cases from children's hospitals emergency departments are weighted no differently than cases from other hospitals. Through this system more than 200000 annual reports are processed representing projected estimates of more than nine million product related injuries per year, categorized by about 800 different generic product codes.

\section{PRELIMINARY CASE SELECTION}

Case selection was aimed at obtaining a reclassified data set that included all NEISS reports involving a baby walker but excluding non-walker case reports. A freedom of information request was made to CPSC for a preliminary data set for the 10 year period 1982-91. The request asked for every report for children less than 24 months of age that included the NEISS product code ' 1508 ' (defined by NEISS as 'baby walkers or jumpers, also consider baby exercisers' ${ }^{18}$ ) in either of the two product code fields ( $n=3829$, unweighted) plus all non-'1508' coded cases that contained the text string 'WALKER' in the 'comments' text field ( $\mathrm{n}=178$, unweighted). The text string inclusion criteria allowed for finding and including non-'1508' coded reports that were baby walker related (false negatives). Excluding reports of cases 24 months of age or older eliminated concern for misclassified geriatric 'walker' related injuries.

False positive reports were identified from the subset of 3829 unweighted reports of injuries to children less than 24 months of age with product code ' 1508 ' by excluding injuries related to 'baby jumpers', 'exercisers', and other non-walker products. Jumper and stroller cases were removed by computer assisted searches for the text strings 'JUMP' or 'STROL' in the event narrative and subsequent manual review. In addition, all reports with a '1508' product code that did not have the text string 'WALK' in the comments field were manually reviewed ( $\mathrm{n}=45$, unweighted) for misspellings or mention of an alternative (nonwalker) juvenile product.

False negative reports were identified by manually reviewing the narratives of the 178 reports (less than 24 months of age) that had the text string 'WALKER' in the comment field 
but were not coded as ' 1508 ' in either product field. A schematic of the case selection procedures is shown in fig 1 . This search strategy fails to identify those presumably few false negatives that misspelled the word 'WALKER' in non- ' 1508 ' coded cases (based on the low rate of misspelling observed within the ' 1508 ' coded cases).

\section{CHILDREN'S HOSPITALS}

It was obvious that the number of child injuries from a certain size sample hospital serving only children must be considerably higher than a similar size non-children's hospital. Yet, CPSC has not performed any special accounting in the NEISS sample for children's hospitals, either by sample stratification or weight adjustment. Because the number of children's hospitals in NEISS could have an important impact on sample estimates, the reclassified and adjusted baby walker reports were analyzed taking into account the number of children's hospitals

\section{Case selection procedures}

Baby walker related reports (unweighted)

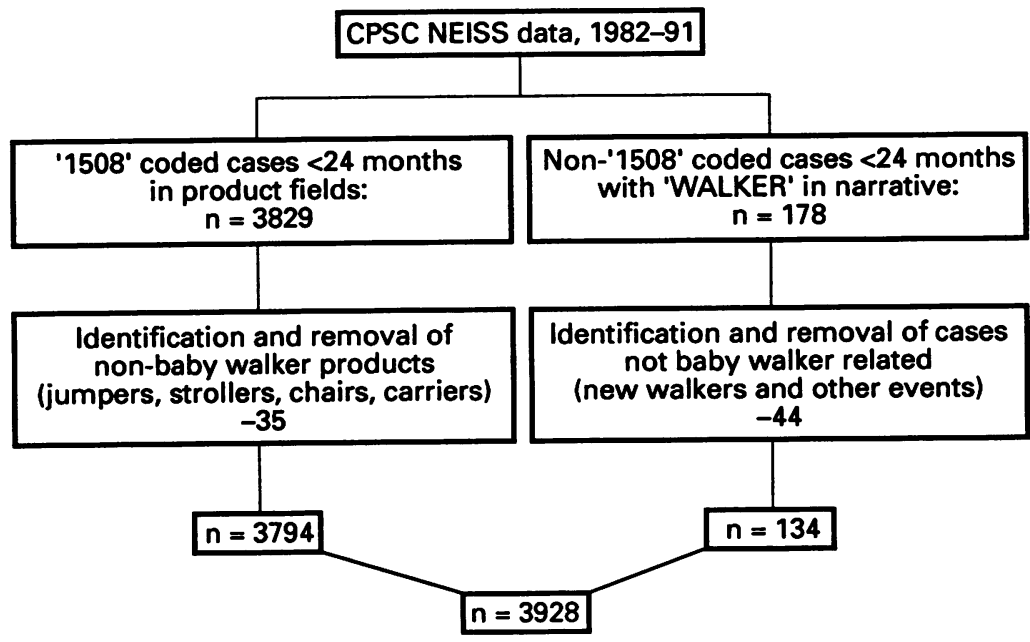

Figure 1 Schematic overview of baby walker case selection and reclassification procedures and results.

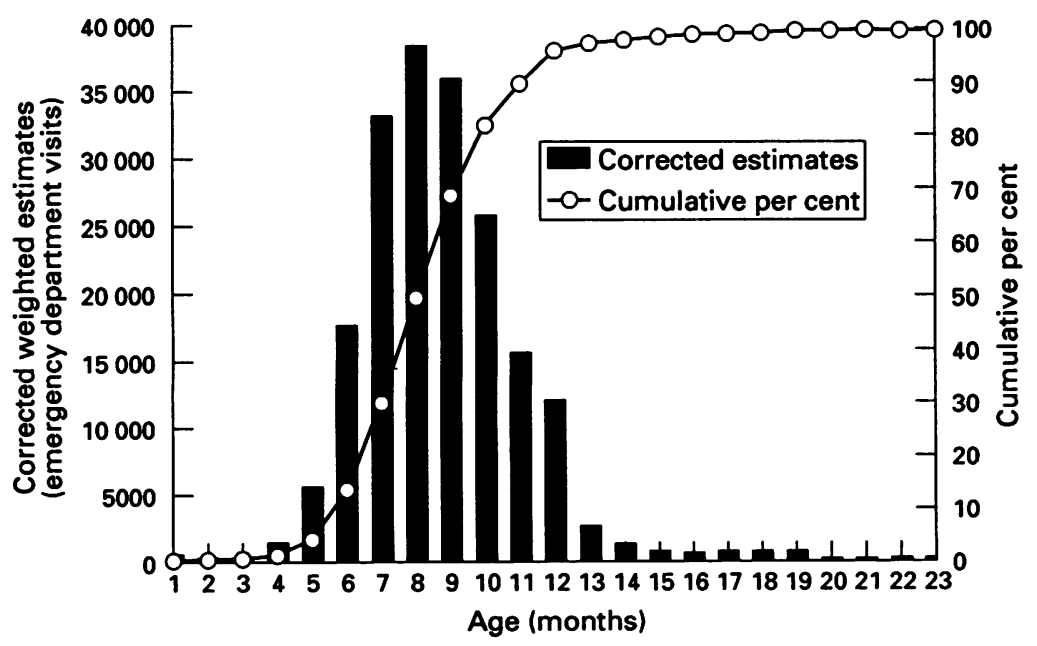

Figure 2 Corrected weighted estimates of baby walker related injuries by age and cumulative incidence reported to CPSC, NEISS, 1982-91. contributing to the data system over different periods. Facility codes for children's hospitals contributing to NEISS were identified from published system descriptions and computer matched to each reported case.

\section{DATA ANALYSIS}

Because changes in the sampling frame between the initial NEISS sample design and subsequent growth of hospital emergency department patient loads used in an updated sample design, CPSC suggests applying an upward adjustment to all weighted estimates for the period 1984 to 1989 by $12 \% .{ }^{19}$ This adjustment was made only for the trend analyses and rates described in the results. Rates were calculated using US live births from 1982 to 1991 as a proxy of exposure. This approximation of the population at risk is appropriate, as most baby walker related injuries occur for a one year period between 4 and 16 months of age (see fig 2).

Sampling errors are measures of the variation in estimates occurring by chance because a sample rather than all emergency departments were surveyed. Generalized NEISS standard errors corresponding to a coefficient of variation of 0.13 and $95 \%$ confidence intervals were applied to the reclassified and rate adjusted data. This coefficient of variation was obtained from CPSC. ${ }^{20}$ It represents the interpolated coefficient of variation for estimates in the range of 10000 to 25000 cases. CPSC computed this coefficient by averaging the generalized sampling errors for annual estimates for the period 1982-92.

\section{Results}

Among the 3829 ' 1508 ' coded reports, 29 were removed because they mentioned jumpers or strollers or had ambiguous narratives.' Two examples of deleted reports were: 'babys jumper fell hitting pt in head. DX: scalp laceration' and 'Pt fell while walking stroller on to floor - fractured clavicle'. Among the 45 reports with ' 1508 ' product codes that did not have the text string 'WALK' in the 'comments' field, 26 of these were obvious misspellings and typographical errors of the word 'WALKER' (for example 'WAKER, WLAKER, etc'), 12 narratives did not mention a juvenile product (for example, 'child fell down stairs'), one report involved a 'BABYCREEPER' stair fall, and these were retained. Six of the 45 reports without the text 'WALK' contained names of (presumed) other types of juvenile products (for example, 'CHAIR, CARRIAGE, CARRIER, CRAWLER') and were excluded, leaving a total of 3794 (unweighted) coded cases.

Among the 178 non-' 1508 ' coded cases with the text string 'WALKER', 41 records contained the text 'NEW WALKER' - referring in all likelihood from the context of the accompanying narratives to a baby just starting to walk, not to the age of a product, and were excluded. Of the remaining 137 reports, 134 consisted of baby walker related cases typified by baby walker related falls, tips, and trips and 
to a lesser extent, baby walker related poisonings, liquid burns, contact burns, and hit by objects. Most of these cases involved more than two products and were coded in NEISS as stair related, stair/rug related, or stair/floor related without a second product code listed. Two typical examples of identified non-' 1508 ' coded cases were (emphasis added): 'Pt fell down a flight of carpeted stairs while in walker landed on carpeted floor head trauma' and 'in a walker pulled on telephone cord which tipped over a crock pot :hot water scalded chest arms wrists hands'. Multiple product involvement was typical of the false negative cases. About $20 \%$ of this subgroup represented instances where NEISS coders did not use the second product code field.

The case selection results are summarized in fig 1. This reclassified (corrected) data set resulted in a projected 10 year population (weighted) total of 195588 estimated cases. This represents an increase of 5158 estimated cases from the unedited ' 1508 ' coded cases. Figure 2 shows the age distribution by month of the reclassified data. Fifty nine per cent of the cases were male. A May-June peak in monthly estimates was observed over the period. Four per cent of the cases were hospitalized.

Figure 3 shows the projected rates by year for the adjusted estimates. The adjustments change the trend data only slightly. The data show an increase in reported baby walker related injury rate over the 10 year period from $4.6 / 1000$ in 1982 to $6.9 / 1000$ in 1991 . However, as can be seen from the graph, with the large $95 \%$ confidence intervals the changes in reported rates never achieve significance.

Regarding children's hospitals, it was deter-

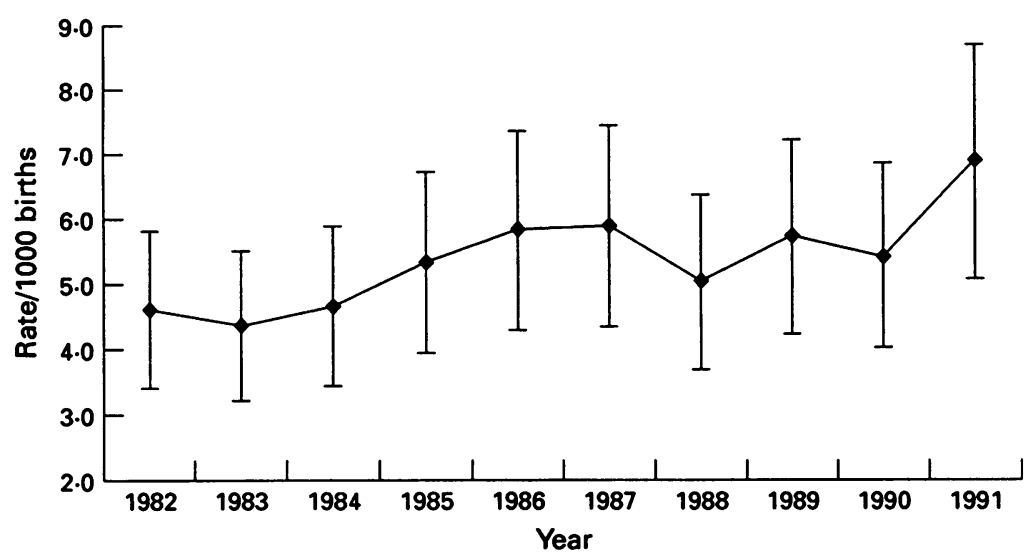

Figure 3 Trends in corrected and adjusted baby walker related injury rates/1000 live births, projected estimates for US 1982-91; 95\% confidence intervals using a 0.13 generalized coefficient of variation are shown. (Source of data: US CPSC, NEISS.)

Children's hospitals contributing to NEISS, US 1982-91

\begin{tabular}{lllll}
\hline City & State & $\begin{array}{l}\text { Emergency department } \\
\text { load stratum }\end{array}$ & First month & Last month \\
\hline Pittsburgh & PA & Large & $1 / 91$ & Current \\
Philadelphia & PA & Very large & $12 / 80$ & Current \\
Miami & FL & Large & $5 / 81$ & $12 / 89$ \\
Fresno & CA & Large & $10 / 78$ & $11 / 89$ \\
Detroit & MI & Large & $12 / 80$ & $9 / 84$ \\
\hline
\end{tabular}

${ }^{\star}$ Large $=25896-42298$ visits, very large $=42299+$. Source: US CPSC mined from CPSC records that as of April 1995, five children's hospitals of different sizes had randomly been a part of the NEISS sample at different times (See table). ${ }^{17}$ In 1991 the baby walker related injury rates at the two children's hospitals in the NEISS sample were 11.9 and $10 \cdot 9 / 10000$ hospital emergency department visits. The overall NEISS baby walker related rate was 2.9/10000 emergency department visits. The two children's hospitals, one large and one very large hospital, reported $14 \%$ of all the NEISS baby walker related injuries though they encompassed $2 \%$ of the patients among NEISS sampled hospitals. In some years, the one very large children's hospital in the sample contributed as much as $17 \%$ of all weighted walker related injury estimates. The new large children's hospital added in 1991 accounted itself for $9 \%$ of the walker related injury estimates for that year; enough to account for over a third $(36 \%)$ of the variation from the year before. The drop in rate from 1989 to 1990 was observed to be about the same as the drop in the percentage of weighted cases contributed by the two children's hospitals that exited the NEISS at the end of 1989.

From a geographic perspective it was noted that both children's hospitals in the 1991 NEISS sample were located in one state and that state (Pennsylvania) had four other hospitals contributing to NEISS. Together, the six Pennsylvania hospitals contributed more than $18 \%$ of all 1991 national walker related injury weighted estimates, though Pennsylvania contains only $3 \%$ of the US infant population.

\section{Discussion}

The preceding analyses clarify baby walker injury misclassification and suggest reasons for the discrepancies in reported estimates of the number of baby walker injuries and the significance of attributed incidence trends. Some misclassification was apparent, with false negatives outnumbering false positives. The net effect of the reclassification was a relatively minor upward correction. No basis for a significant impact of 'jumper' related cases in the ' 1508 ' coded data was found. The narrative text string contained in each record was crucial to determining the magnitude and direction of misclassification.

Adjustments for birth rates over the study period resulted in relatively minor downward corrections in rates for most recent years as births generally increased over the study period. The largest uncertainties in the trend estimates stem not from misclassification or rate adjustments but from the relatively small NEISS sample size resulting in large standard errors and from hospital type and geographic imbalance in the actual random sampling selection process. On sample variation grounds alone, about a $70 \%$ increase in observed rates from 4.6 to 7.9 would be necessary to achieve significance at the $\alpha=0.05$ level from one period to another. There is a trend toward an increase in rates, but it is not significant at the $\alpha=0.05$ level. 
The drop in calculated rates from 1989 to 1990 and the large increase in the reported rate from 1990 to 1991 (and likely to be carried through for estimates to the present) can be attributed, in part, to the changes in the underlying NEISS sample selection process as children's hospitals went out of and into the sample. While the contributions of the children's hospitals do not explain all the variation, they are an important factor in year to year changes. The impact on trends described due to hospital type probably holds for other children's product related injuries reported to NEISS. This effect will be even greater for products that are used more in north eastern climates such as ice skates, snow skis, and sleds because both children's hospitals now reporting to NEISS happen to be located in colder climates (both are in Pennsylvania). Conversely, child product related injuries that involve more temperate activities (outdoor sports and in-line skating for example) may be underestimated.

In early 1995, the CPSC began an effort to collect reports from nine additional children's hospitals to enhance the reporting of child product related injuries. Those data will be used for case finding purposes the first year, but CPSC plans to include those children's hospitals in producing national estimates in the future (Arthur McDonald, CPSC, personal communication, 20 November 1995). It was recognized that a probability sample of children's hospitals cannot be used to create national estimates for children's injuries seen in all emergency departments.

Other forms of bias that can occur in a sample surveillance system such as NEISS were not examined in this study. Most of the information in NEISS represents third party reporting subject to varying biases. If the emergency department documentation is in error (by omission or commission) or the walker relatedness of the patient's injury was undocumented in the medical record, then adjusted estimates will be affected accordingly. Another factor potentially affecting estimates was exposure to the risk. Denominators used for risk calculations should reflect the combined exposure to and use of the product. However, information on the rate of baby walker ownership and the length and frequency of use was not available.

The effect of changes in multistory housing on exposure risk over time is not known. According to the CPSC, 'multistory housing has risen dramatically, from about 39 percent of all new single family housing in 1980 to about 52 percent in $1989^{\prime} .{ }^{16}$ The regional pattern of this increase is obscure since NEISS baby walker injury reports are not geographically randomly distributed and the proportion of all housing that is multistory over time (a large increase in a small factor will show little effect) is unknown. Any effect of increased multistory housing may be muted by the large proportion of baby walker stair falls that occur down basement stairs, not second story to first story falls. The issue of consistently proportional representation of trauma centers in the NEISS sample was not looked at either, but this could theoretically impact on the stability of estimated hospitalization rates for various products over time.

\section{Conclusions and recommendations}

By clarifying appropriate case selection criteria using specific product codes and narrative text strings, through rate adjustments and calculations of sample variances, and by examining the types and locations of hospitals contributing to the product specific sample over time, important NEISS limitations have been portrayed.

Misclassification in NEISS baby walker reports is minimal with false negatives outweighing false positives. A $1 \%$ false positive rate was countered by a $4 \%$ false negative rate. The short narrative text string proved to be a valuable adjunct to the coded data. For trend analysis of product related injuries at the level of occurrence studied for baby walkers, NEISS suffers from poor sensitivity due to relatively large sampling error. Year to year, walker related injury trend data can be affected by children's hospitals coming in and out of the sample. For all children's injuries, NEISS' hospital type sampling scheme reflects a random geographic imbalance from 1991 to 1994 because one north eastern state contributes both of the reporting children's hospitals.

To overcome these problems, it is recommended that NEISS promotes consistent second product coding and improve documentation and coder training to ensure completion of all product fields in multiple products related injury events. Although few jumper injuries were included in the ' 1508 ' coded cases, it would be prudent to add a new separate code to allow differential (unique) coding for baby walkers and jumpers. The CPSC should ensure the stability and representation of children's hospitals in the NEISS sample by stratifying children's hospitals in the sample design. Most importantly, the CPSC should enlarge the hospital sample size to reduce sample variation, though this will require additional cost. Without these changes, uncertainty will continue to accompany the use of NEISS data for interpreting incidence and trend patterns for baby walker related injuries in particular, and possibly for other product related child injuries.

Parts of this paper were presented at the Annual Meeting of the American Public Health Association, in San Francisco, October 1993.

The author wishes to thank the helpful and thorough reviews of this manuscript by Dr Jeffrey Coben, Dr Herbert Garrison, Dr Samuel Forjuoh, Mr Steve Dearwater, and Mr Arthur McDonald. I would also like to thank the Allegheny County McDonald. I would also like to thank the Allegheny County
Health Department and Dr Mary Jones for assistance in Health Department and Dr Mary Jones for assistance in
procuring the NEISS data and CPSC and NEISS hospitals for supplying the data upon which this work was based.

1 US Consumer Product Safety Commission. NEISS data highlights. Vol 10. Washington, DC: US CPSC, Jan-Dec 1986.

2 Schieber R, Branche-Dorsey C, Ryan G. Comparison of in-line skating injuries with rollerskating and skateboarin-line skating injuries with rollerskating
ding injuries. $\mathscr{f} A M A$ 1994; 271: $1856-8$.

3 Reilly J, Walter $M$. Consumer product aspiration and ingestion in children: analysis of emergency room reports to the national electronic injury surveillance system. Ann Otol Rhinol Laryngol 1992; 101: 739-41.

4 Senturia Y, Binns H, Christoffel K, Tanz R. Exposure corrected risk estimates for childhood product related injuries. Accid Anal Prev 1993; 25: 473-7. 
5 US Consumer Product Safety Commission. News from CPSC-1995, March [Online]. Available Gopher: gopher://cpsc.gov:70/11/Pre_Rel/Pre_Rel/95_Pre (18 gopher://cpsc.

6 US Consumer Product Safety Commission. Proposed rules. [Online]. Available Gopher: gopher://cpsc.gov:70/11/ [Online]. Available Gopher: gopher://cpsc.gov:

7 Reider M, Schwartz C, Newman J. Patterns of walker use and walker injury. Pediatrics 1986; 78: 488-93.

8 Coury D, Kasten E, Shepherd L, and the Columbus PROBE Group. Infant walker use in private practice populations. Am $\mathcal{f}$ Dis Child 1992; 146: A507.

9 Eskind A. Bringing up baby. Wall Street fournal, 13 Apri 1993.

10 Marcella S, McDonald B. The infant walker: an unappreciated household hazard. Conn Med 1990; 54: 127-9.

11 American Medical Association Board of Trustees. Use of infant walkers. Am F Dis Child 1991; 145: 933-4.

12 US Consumer Product Safety Commission. Stair steps and baby walkers don't mix. Consumer Product SAFETY ALERT (No 009207) Washington, DC: US CPSC, 1992.

13 Partington M, Swanson J, Meyer F. Head injury and the use of baby walkers: A continuing problem. Ann Emerg Med

4 Gaudreault P, McCormick M, Lacouture P, Lovejoy F.
Poisoning exposures and use of syrup of ipecac in children less than 1 year old Ann Emerg Med 1986; 15: 808-10. 15 GRACO Children's Products. Comments opposing the petition of the Consumer Federation of America et al, to ban tion of the Consumer Federation of America et al, to ban baby walkers. (Address to the commission.) (Written testimony submitted to the Consumer Product Safety Commission). Washington, DC: US CPSC, 4 December 1992.

16 US Consumer Product Safety Commission. Baby walker petition, HP 92-2. (Briefing package) Washington, DC: US CPSC, Released March, 1993.

17 US Consumer Product Safety Commission, National Injury Information Clearinghouse. List of NEISS hospitals. Washington, DC: US CPSC, April 1995.

18 US Consumer Product Safety Commission. NEISS coding manual. Washington, DC: US CPSC, 1987.

19 US Consumer Product Safety Commission. Memo from Leonard Schachter to Terrance R Karels regarding petition requesting a ban of baby walkers (HP 92-2). Washington, DC: US CPSC, 10 December 1992: 4,5. (Part of baby walker petition briefing package HP 92-2.)

20 US Consumer Product Safety Commission. The NEISS sample (design and implementation). Washington, DC: CPSC Division of Hazard and Injury Data Systems, February, 1994.

\section{Canadian daredevil seeks tightrope record}

From Reuters, Hong Kong: 'a man who dubs himself Canada's "prince of the air" will attempt the longest and highest tightrope walk in history tomorrow above the torrents of China's awesome Three Gorges on the Yangtze River. With no margin for error, Jay Cochrane admits to fear.

Only a balancing pole and determination will keep him on the wire. On either side of about 600 metres of wire stretched across the narrowest point of the gorge is a sheer rock face dropping 400 metres. "Of course I'm afraid," he said yesterday. Speaking by telephone from China, Cochrane said: "I have a healthy respect and fear of what I'm about to attempt".

The 54 year old Cochrane ... has performed death defying stunts for 40 years. He holds the Guinness Book of Records title for living on a wire for 21 days and nights. And, if successful tomorrow, Cochrane will shatter the current 500-metre tightrope walking record. "I'm not a stuntman", he said "I'm taking a calculated risk".' (Editor's note: but are such calculated risks good examples for children?)

\section{Along the same lines: in-line skating role models?}

A contributor to CCSN's BBS writes: 'As an in-line instructor, one of the toughest parts of my job is trying to get skaters, be they adults or children, to wear protective gear. While on vacation in BC I had an opportunity to meet Ani, a professional in-line skater who has appeared in aggressive movies and magazines. When Ani skates he wears only a knee brace. He told me that he comes to in-line through skateboarding and skateboarders generally skate without gear.

Ani does have cat-like grace even when he falls; after watching him skate I can attest to that. However, in my opinion, this is what is wrong with my sport. The pro skaters either don't realize or forget that once they grace the pages of a magazine or appear in a movie they become more than a skater - they become role models. Ani is an adult and realizes the risks he takes. What worries me are the kids who buy the magazines, who live for the movies, but who are neither lucky nor born with Ani's skills. 